Just as with morbid jealousy, with which it has many parallels, morbid remorse can have a delusional, or real but overvalued, precipitating cause, or may also not have any cause which the patient is able to articulate. For example, it is not uncommon for a depressed elderly patient to feel that they have ruined the lives of their children. Their preoccupation and distress is with the consequence of their imagined short-comings and is therefore better described as a morbid remorse, than as a sense of guilt for being an imperfect parent, a guilt which probably all parents would acknowledge. To say that they are "filled with remorse" conveys a more empathic understanding of their mental state than to suggest that they are "experiencing feelings of guilt".

Regional Treatment Centre (O)

G. N. CONACHER

P.O. Box 22

Kingston, Ontario

K7L 4V7

Availability of the Hospital Anxiety and Depression (HAD) Scale

SIR: In recent issues, Rice \& Donnelly (Psychiatric Bulletin, June 1992, 16, 329-330) found that the HAD Scale was used in clinical work by consultant psychiatrists, and Tyrer (Journal, June 1992, 160, 733-741) recommended its use in the assessment of pain. I welcome the opportunity to inform readers of its present availability. Soon after publication of the study introducing the HAD it was made freely available by the good offices of the Medical Liaison Service of Upjohn. After some years that service had to cease. The HAD is now produced in convenient single-sheet format with inbuilt scoring device, together with a chart for successive recording of scores in order to indicate progress under treatment. For bulk supply of this material a charge is necessary to cover costs; alternatively, single copies may be obtained and personal copies made by the user.

Despite the word 'hospital', the HAD may be used in community work. It is acceptable to and easily completed by the patient. It provides a distinction between the two concepts of depression and anxiety; the depression subscale is largely a reflection of anhedonia since this state seems to be the best clinical marker of the aspect of depression indicating disturbed neurobiological mechanisms (Snaith, 1992). The HAD has been translated into most European languages, also into Arabic, Israeli, Urdu, Japanese, and Chinese, and other translations are being considered.
Those wishing for information should send a stamped addressed envelope (A4 size) to the address below. A bibliography of studies validating the HAD in different settings will be included; in order to keep this up-to-date I should be pleased to receive reprints of all studies incorporating the use of the HAD.

SNaIth, R. P. (1992) Anhedonia. British Medical Journal (in press).

Academic Unit of Psychiatry

R. P. SNAITH

Clinical Sciences Building

St James's University Hospital

Leeds LS9 7TF

\section{Fingerprints and statistics}

SIR: I read with interest the paper by Mellor on dermatoglyphics and schizophrenia (Journal, April 1992, 160, 467-472). The author remarks that many of the studies involving dermatoglyphics have yielded ambiguous results (p. 467).

Some of this ambiguity may be due to the incorrect use of the $\chi^{2}$ test, where it is assumed that all observations are independent. Mellor (1968) analysed fingerprint patterns in schizophrenics and normal individuals with the pattern on a finger as the unit of observation (hence 500 individuals yield 5000 observations). However, pattern types on the fingers are not statistically independent. From Table 3 of Dr Mellor's recent paper it can be seen that around $80 \%$ of homologous fingers have the same pattern type!

Donner \& Donald (1988) have proposed an adjusted $\chi^{2}$ statistic which can be used when each individual provides several associated observations. They show that the results obtained from an analysis which ignores the dependency between observations are likely to be more statistically significant than those obtained from an analysis which takes this dependence into account. Thus statistically significant findings from the analysis of fingerprint patterns as independent observations may be misleading.

DONner, A. \& Donald, A. (1988) The statistical analysis of multiple binary measurements. Journal of Clinical Epidemiology, 41, 899-905.

Mellor, C. S. (1968) Dermatoglyphics in schizophrenia. Part I: Qualitative aspects. Part II: Quantitative study. British Journal of Psychiatry, 114, 1387-1397.

\author{
Department of Public Health Medicine \\ UMDS (Guy's Campus) \\ London SEI 9RT
}

